\title{
Space-Time Transformation in Flux-form Semi-Lagrangian Schemes
}

\author{
Peter C. Chu* and Chenwu Fan \\ Naval Ocean Analysis and Prediction Laboratory, Department of Oceanography, \\ Naval Postgraduate School, Monterey, CA 93943, USA
}

Received 11 June 2008, accepted 25 May 2009

\begin{abstract}
With a finite volume approach, a flux-form semi-Lagrangian (TFSL) scheme with space-time transformation was developed to provide stable and accurate algorithm in solving the advection-diffusion equation. Different from the existing fluxform semi-Lagrangian schemes, the temporal integration of the flux from the present to the next time step is transformed into a spatial integration of the flux at the side of a grid cell (space) for the present time step using the characteristic-line concept. The TFSL scheme not only keeps the good features of the semi-Lagrangian schemes (no Courant number limitation), but also has higher accuracy (of a second order in both time and space). The capability of the TFSL scheme is demonstrated by the simulation of the equatorial Rossby-soliton propagation. Computational stability and high accuracy makes this scheme useful in ocean modeling, computational fluid dynamics, and numerical weather prediction.
\end{abstract}

Key words: TFSL scheme, Flux-form semi-Lagrangian scheme, Characteristic line, Advection-diffusion equation, Finite volume, Conservative finite difference, Equatorial Rossby soliton.

Citation: Chu, P. C. and C. Fan, 2010: Space-time transformation in flux-form semi-Lagrangian schemes. Terr. Atmos. Ocean. Sci., 21, 17-26, doi: 10.3319/ TAO.2009.05.25.01(IWNOP)

\section{INTRODUCTION}

From a physical point of view, advection of a passive tracer is the simple transition of a quantity without diffusion and dispersion. Numerical approaches in atmospheric and oceanic modeling inevitably introduce diffusion (or dissipation) and dispersion into the approximate solution. The numerical diffusion and dispersion are aliens to the process that is being modeled (Chu and Fan 1998, 1999). As applied to a constituent advection problem, these numerical artifacts manifest themselves as nonphysical mixing by numerical diffusion, nonphysical highs and lows in the constituent field caused by dispersion, and nonphysical tracer spectra caused by trapping in nonpropagating small spatial scales (Rood 1987). For example, the commonly used upwind scheme is conditionally stable (with the Courant number being much smaller than 1) and some artificial viscosity is introduced. Hence, less the numerical diffusion and dispersion errors equates to better model performance.

Many numerical algorithms have been proposed to reduce numerical diffusion and dispersion errors and to keep

\footnotetext{
* Corresponding author

E-mail:pcchu@nps.edu
}

the numerical stability. The flux-form semi-Lagrangian scheme is among them. Using the flux-form semi-Lagrangian schemes, artificial viscosity is reduced and stability is kept without the limitation of a Courant number (Casulli 1990, 1999). In this study, we use a finite volume approach to develop time-space transformed flux-form semi-Lagrangian (TFSL) scheme. This scheme has an explicit form and much less diffusion and dispersion errors.

The stability and accuracy of numerical schemes for ocean models are usually verified using the propagation of a Rossby soliton on an equatorial beta-plane. In principle, the soliton propagates to the west at a fixed phase speed, without a change of shape. Since the uniform propagation and shape preservation of the soliton are achieved through a delicate balance between linear wave dynamics and nonlinearity. In other words, the Rossby soliton is non-diffusive and non-dispersive (Boyd 1980), which makes it a perfect test case for verification of numerical schemes in ocean models since any diffusion and dispersion in the numerical solution of the Rossby soliton are computational errors. Interested readers are referred to the website: http://marine. rutgers.edu/po/index.php?model=test-problems. 
To show the benefit of using the TFSL scheme, we first show instability and large diffusion and dispersion errors in numerical solution of the Rossby soliton using the existing schemes such as the flux-form upwind, flux-form central, Lax-Wendroff, and flux-form semi-Lagrangian schemes. Then, we will describe the procedures of the TSFL scheme development and verification. The rest of paper is organized as follows. Section 2 describes the equatorial Rossby soliton and its usefulness for an ocean model verification. Section 3 shows the failure of the three existing schemes (upwind, central, Lax-Wendroff, semi-Largangian) in simulating the equatorial Rossby soliton. Section 4 introduces the TFSL scheme. Section 5 derives the analytical form of the amplification factor of the TFSL-scheme. Section 6 shows the capability of the TFSL-scheme in simulating the equatorial Rossby soliton. Section 7 presents our conclusions.

\section{ROSSBY SOLITON}

Let $\Omega$ be the angular frequency of earth's rotation and $R$ be the earth radius, and let $(x, y)$ be the spatial coordinates with unit vectors $(\mathbf{i}, \mathbf{j})$ and $t$ be the time. Consider a single layer of homogeneous ocean layer with depth of $H$. Lamb's parameter is defined by

$E=\frac{4 \Omega^{2} R^{2}}{g H}$

where $g$ is the gravitational acceleration. The length and time are non-dimensionalized by

$L=\frac{R}{E^{1 / 4}}, \quad T=\frac{E^{1 / 4}}{2 \Omega}$

For the mean ocean depth $H=4 \mathrm{~km}$, the earth radius $R=$ $6370 \mathrm{~km}$, and $\Omega=2 \pi /(86400 \mathrm{~s})$, the length and time scale are $L=543 \mathrm{~km}, T=22.39 \mathrm{hr}$. Let $(x, y)$ be the non-dimensional Cartesian coordinates, $(u, v)$ be the non-dimensional velocity components in the meridional and latitudinal directions, and $\phi$ be the non-dimensional surface elevation. After defining

$\mathrm{s} \equiv x-c t$

and transforming the nonlinear shallow water wave equations into a frame of reference moving with the linear wave, the flow variables $(u, v, \phi)$ for the mode-1 wave can be represented by (Boyd 1980)

$u(s, y, t)=\frac{\left(6 y^{2}-9\right)}{4} \eta(s, t) \exp \left(-\frac{y^{2}}{2}\right)$

$$
\begin{aligned}
& v(s, y, t)=2 y \frac{\partial \eta(s, t)}{\partial s} \exp \left(-\frac{y^{2}}{2}\right) \\
& \phi(s, y, t)=\frac{\left(6 y^{2}+3\right)}{4} \eta(s, t) \exp \left(-\frac{y^{2}}{2}\right)
\end{aligned}
$$

and the variable $\eta(s, t)$ satisfies

$$
\begin{aligned}
\frac{\partial \eta}{\partial t}-f_{1} \eta \frac{\partial \eta}{\partial s}-f_{2} \frac{\partial^{3} \eta}{\partial s^{3}} & =0 \\
f_{1}=1.5366, f_{2} & =0.098765
\end{aligned}
$$

which is the Korteweg-de Vries (KDV) equation with the exact solution,

$$
\begin{aligned}
\eta(s, t)= & A \operatorname{sech}^{2}\left[B\left(s+\mu B^{2} t\right)\right] \\
& A=0.772 B^{2}, B=0.394, \mu=0.395
\end{aligned}
$$

Substitution of the exact solution Eq. (6) into the third term in the lefthand side of Eq. (5) leads to

$$
\frac{\partial \eta}{\partial t}-f_{1} \eta \frac{\partial \eta}{\partial s}=S
$$

$S=f_{2} \frac{\partial^{3} \eta}{\partial s^{3}}$

where $S$ is treated as a source/sink term. Evidently Eq. (7) has the analytical solution Eq. (6). Since the analytical solution Eq. (6) exists, the Rossby soliton Eq. (7) is a perfect test case for verifying the stability and accuracy of numerical schemes since the diffusion term has been changed into the given source/sink term. To do so, the fluid is assumed to occupy the equatorial region surrounding the earth. The zonal direction is discretized into 120 cells (i.e., resolution at $3^{\circ}$ longitude). The increment $\Delta s$ is given by

$$
\Delta s=\frac{2 \pi R}{120 L} \approx 0.256
$$

The independent variables $(s, t)$ are discretized by $s_{i}=s_{i-1}+$ $\Delta s, t^{n}=t^{n-1}+\Delta t, i=1,2, \ldots ; n=1,2, \ldots .$, with $\Delta t$ the time step. The dependent variable $(\eta)$ at $\left(s_{i}, t^{n}\right)$ is represented by $\eta_{i}^{n} \equiv \eta\left(s_{i}, t^{n}\right)$.

\section{SEVERAL EXISTING SCHEMES}

Equation (7) can be discretized using the flux-form upwind scheme, 
$\eta_{i}^{n+1}=\eta_{i}^{n}+\frac{f_{1} \Delta t}{2 \Delta x}\left[\left(\eta_{i+1}^{n}\right)^{2}-\left(-\eta_{i}^{n}\right)^{2}\right]+Q_{i}^{n}$

the flux-form central scheme,

$\eta_{i}^{n+1}=\eta_{i}^{n}+\frac{f_{1} \Delta t}{2 \Delta x}\left[\left(\eta_{i+1 / 2}^{n}\right)^{2}-\left(\eta_{i-1 / 2}^{n}\right)^{2}\right]+Q_{i}^{n}$

the Lax-Wendroff scheme,

$\eta_{i}^{n+1}=\eta_{i}^{n}-\frac{C_{i}^{n}}{2}\left(\eta_{i+1}^{n}-\eta_{i-1}^{n}\right)+\frac{\left(C_{i}^{n}\right)^{2}}{2}\left(\eta_{i+1}^{n}-2 \eta_{i}^{n}+\eta_{i-1}^{n}\right)+Q_{i}^{n}$

and the semi-Lagrangian scheme,

$\eta_{i}^{n+1}=\eta_{i}^{n}+\frac{f_{1} \Delta t}{2 \Delta x}\left(\eta_{i+1 / 2}^{* 2}-\eta_{i-1 / 2}^{* 2}\right)+Q_{i}^{n}$

with

$Q_{i}^{n} \equiv \int_{t^{n}}^{t^{n+1}} S\left(s_{i}, t\right) d t=\frac{2 A f_{2}}{\mu}\left[2 \sec h^{2}(Y)-3 \sec h^{4}(Y)\right]_{t^{n}}^{n+1}$

$C_{i}^{n} \equiv \frac{f_{1} \eta_{i}^{n} \Delta t}{\Delta s}, \quad Y \equiv B\left(s+\mu B^{2} t\right)$

$\eta_{i+1 / 2}^{* 2} \equiv \frac{1}{2}\left[\left(\eta_{i+1 / 2}^{n}\right)^{2}+\left(\eta_{i+1 / 2}^{n+1}\right)^{2}\right], \quad \eta_{i+1 / 2}^{n+1} \equiv \eta_{i+1 / 2-C_{i+1 / 2}^{n}}^{n}$

In order to compare the difference between numerical and exact solutions (a westward propagating Rossby soliton), the zonal equatorial strip is assumed to be infinitely long. When the Rossby soliton travels over $n \times 120$ cells, it goes around the earth once $n$ times (called $n$ cycles). The exact solution at $t=0$ is taken as the initial condition,

$\eta=(s, 0)=A \sec h^{2}(B s)$

with $s=0$ denoting $0^{\circ}$ longitude.

Three difference Eqs. (10) - (12) are solved numerically from the initial condition Eq. (15) representing the upwind, central, and Lax-Wendroff schemes (Lax and Wendroff $1960)$ with varying $\Delta t$ at each time step for a given Courant number $(C=0.75), \Delta t \equiv \frac{C \Delta s}{f_{1} \max \left(\left|\eta_{i}^{n}\right|\right)}$. Selection of $C=0.75$ is due to the fact that the proposed TFSL scheme will be reduced to the Lax-Wendroff scheme for $C \leq 0.5$ [see Eq. (36)]. After obtaining the numerical solution, $\eta\left(x_{i}, t_{n}\right)$, substituting it into Eq. (4c) yields $\phi\left(s_{i}, y_{j}, t_{n}\right)$. The accuracy of the schemes can be verified through their capability in predicting the westward propagation of the Rossby soliton. To do so, the surface elevation $\phi\left(s_{i}, y_{j}, t_{n}\right)$ is plotted with contour values of $2.13,4.26,6.4,8.53,10.66,12.79,14.93$, and $17.06 \mathrm{~cm}$. All the numerical schemes greatly distort the Rossby soliton (Fig. 1). When the ratio of the root-mean square error versus the root-mean of the analytical solution is greater than $100 \%$, the numerical solution is considered divergence. Figure 1 shows that the numerical solution diverges at $27^{\circ} 45^{\prime} \mathrm{W}$ using the flux-form central scheme, at $54^{\circ} 45^{\prime} \mathrm{W}$ using the Lax-Wendroff scheme, and at $30^{\circ} \mathrm{W}$ af ter one cycle around the earth using the flux-form upwind scheme. Comparing Figs.1b - d to Fig. 1a, the numerical solutions are totally different from the analytical solution.

\section{TFSL-SCHEME}

\subsection{Semi-Lagrangian Method}

Consider the advection of a passive scalar $\phi(\mathbf{x}, t)$ by the velocity $\mathbf{u}(\mathbf{x}, t)$. The Eulerian formulation is given by

$\frac{D \phi}{D t} \equiv \frac{\partial \phi}{\partial t}+\mathbf{u} \cdot \nabla \phi=S$

where $\mathbf{x}$ is the position vector, $D / D t$ denotes the material derivative, while the Lagrangian counterpart is

$\frac{d \phi_{p}}{d t}=S, \quad \frac{d \mathbf{x}_{p}}{d t}=\mathbf{u}\left(\mathbf{x}_{p}, t\right)$

where the subscript ' $p$ ' shows the fluid particle in Lagrangian sense. Although Eqs. (16) and (17) carry the same physical information, their discretization and numerical implementation is different: Eq. (16) is discretized on an Eulerian grid with a finite number of grid points and then time-advanced, while Eq. (17) is integrated for a finite number of fluid particles.

Semi-Lagrangian methods combine both Eulerian and Lagrangian points of view; the scalar field is discretized on an Eulerian grid, but is advanced in time using Eq. (17). The key element in accomplishing this is the identification of each grid point $\mathbf{x}_{i}$ as the arrival point, for instance, at $t+\Delta t$, of a particle originating from $\mathbf{x}_{i}^{*}$ at time $t$. The algorithm has three steps: (a) The particle associated with each grid point $\mathbf{x}_{i}$ at time $t+\Delta t$ is traced back to its location $\mathbf{x}_{i}^{*}$ at time $t$,

$\mathbf{x}_{i}^{*}=\mathbf{x}_{i}-\int_{t}^{t+\Delta t} \mathbf{u}(\tau) d \tau$

(b) The scalar value at $\left(\mathbf{x}_{i}^{*}, t\right)$ is obtained by interpolating the known values at neighboring grid points,

$\phi\left(\mathbf{x}_{i}^{*}, t\right)=P\left[\phi\left(\hat{x}_{i k}\right), t\right]$ 
where $P$ is any interpolation operator and $\left(\hat{x}_{i k}\right)$ denotes the set of interpolation points associated with $\mathbf{x}_{i}^{*}$, for example, the nodes of the cell containing $\mathbf{x}_{i}^{*}$; (c) Finally, the scalar is updated,

$\phi\left(\mathbf{x}_{i}, t+\Delta t\right)=\phi\left(\mathbf{x}_{i}^{*}, t\right)+Q_{i}$

Thus, the main issues of the semi-Lagrangian method are the backward integration in step (a) and the interpolation in step (b).

\subsection{Flux Form}

Equation (16) can be rewritten in the flux form with inclusion of diffusion,

$$
\frac{\partial \phi}{\partial t}=\nabla \cdot \mathbf{F}+S, \quad \mathbf{F}=-\mathbf{u} \phi+\kappa \nabla \phi
$$

where $k$ is the diffusion coefficient. Let the dependent variable $\phi(\mathbf{x}, t)$ be defined on the space $\Omega, 0 \leq x \leq L_{x}, 0 \leq y \leq$ $L_{y}, 0 \leq z \leq L_{z}$.

with $\left(L_{x}, L_{y}, L_{z}\right)$ the lengths in $(x, y, z)$ directions. Let $\Delta x=\frac{L_{x}}{N_{x}}, \Delta y=\frac{L_{y}}{N_{y}}, \Delta z=\frac{L_{z}}{N_{z}}$ be the uniform spatial increments with $\left(N_{x}+1, N_{y}+1, N_{z}+1\right)$ the grid numbers. Integrating Eq. (21) for the finite volume, $\Delta \Omega_{i j k}=\left[x_{i-1 / 2} \leq x \leq x_{i+1 / 2}\right.$, $\left.y_{j-1 / 2} \leq y \leq \mathrm{y}_{j+1 / 2}, z_{k-1 / 2} \leq z \leq z_{k+1 / 2}\right], x_{i \pm 1 / 2} \equiv x_{i} \pm \frac{\Delta x}{2}, y_{j \pm 1 / 2}$ $\equiv \boldsymbol{y}_{j} \pm \frac{\Delta y}{2}, z_{k \pm 1 / 2} \equiv \mathrm{z}_{k} \pm \frac{\Delta z}{2}$, from $t_{n}$ to $t_{n+1}=t_{n}+\Delta t$, we obtain the finite difference equation of the flux-averaged transport,

(a) Analytic Solution with Courant Number $=0.75$

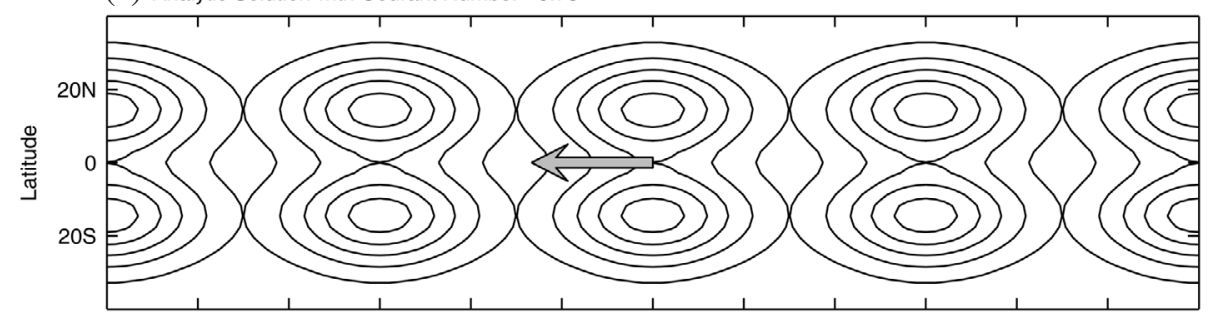

(b) Up Wind Scheme One Cycle

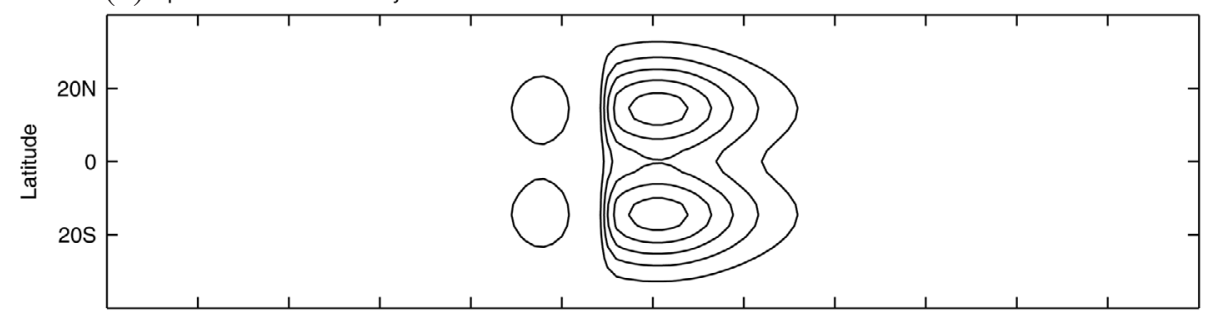

(c) Centeral Explicit Scheme diverges at lon: $27.75 \mathrm{~W}$

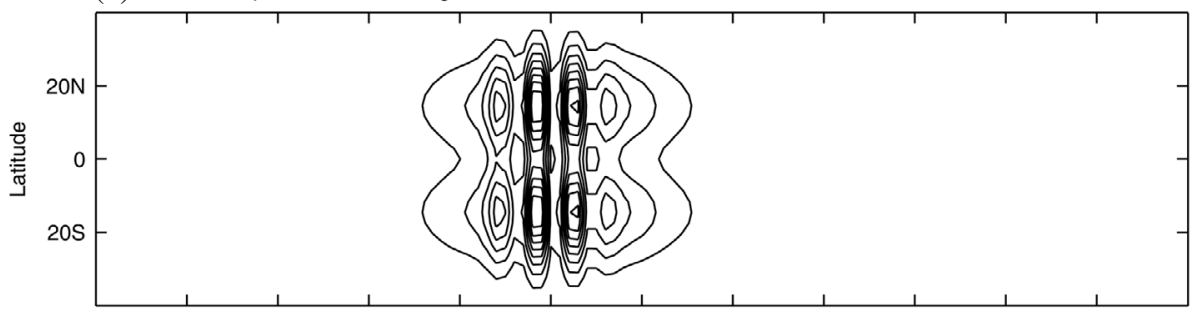

(d) Lax-Wendroff Scheme diverges at lon: $54.75 \mathrm{~W}$

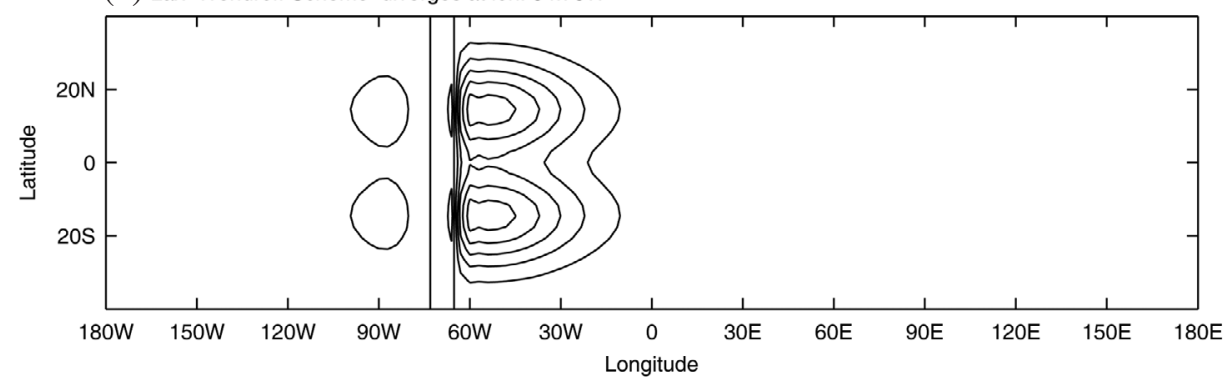

Fig. 1. Surface elevation $\phi(s, y, t)$ of the Rossby solitons obtained from an (a) exact solution, and numerical integration with $C=0.75$ using the (b) flux-form upwind scheme, (c) flux-form central scheme, and (d) Lax-Wendroff scheme. Note that the numerical solution diverges at $30^{\circ} \mathrm{W}$ after one cycle using the flux-form upwind scheme, at $27^{\circ} 45^{\prime} \mathrm{W}$ using the flux-form central scheme, and at $54^{\circ} 45^{\prime} \mathrm{W}$ using the Lax-Wendroff scheme. 


$$
\begin{aligned}
\frac{\tilde{\phi}_{i, j, k}^{n+1}-\tilde{\phi}_{i, j, k}^{n}}{\Delta t}= & \frac{\bar{F}_{i, 1 / 2, j, k}^{(n, n+1)}-\bar{F}_{i-1 / 2, j, k}^{(n, n+1)}}{\Delta x}+\frac{\bar{G}_{i, j+12, k}^{(n, n+1)}-\bar{G}_{i, j-12, k}^{(n, n+1)}}{\Delta y} \\
& +\frac{\bar{H}_{i, j, k+1 / 2}^{(n, n+1)}-\bar{H}_{i, j, k-1 / 2}^{(n, n+1)}}{\Delta z}+\hat{S}_{i, j, k}
\end{aligned}
$$

where $(F, G, H)$ are components of the vector $\mathbf{F}$, and

$\overline{\mathbf{F}}^{(n, n+1)}=\frac{1}{\Delta t} \int_{t_{n}}^{t_{n+1}} \mathbf{F} d t$

represents the temporal average (from $t_{n}$ to $t_{n+1}$ ). The tilde represents the volume average over $\Omega_{i j k}$,

$\tilde{\phi}_{i j k}=\frac{1}{\Delta x \Delta y \Delta z} \iiint_{\Omega_{j j k}} \phi d x d y d z$

The hat represents the combined volume $\left(\Omega_{i j k}\right)$ and temporal average (from $t_{n}$ to $t_{n+1}$ ),

$\hat{S}_{i j k}=\frac{1}{\Delta t \Delta x \Delta y \Delta z} \int_{t_{n}}^{t_{n+1}} \iiint_{\Omega_{j i k}} S d x d y d z d t$

For the finite volume $\Delta \Omega_{i j k}$, the flux at $x=x_{i-1 / 2}$ and $t=t_{n}$ is calculated by

$F_{i-1 / 2, j, k}^{n}=\frac{1}{\Delta y \Delta z} \int_{z k-1 / 2}^{z_{k+112}} \int_{y_{j-1 / 2}}^{y_{j+1 / 2}}\left(\kappa \frac{\partial \phi}{\partial x}-u \phi\right)_{x=x_{i-1 / 2}}^{t=t_{n}} d y d z$

To solve Eq. (22) numerically, we need to compute the temporally integrated fluxes, $\bar{F}_{i+112, j, k}^{(n, n+1)}, \bar{F}_{i=12, j, k}^{(n, n+1)}, \bar{G}_{i, j+12, k}^{(n, n+1)}, \bar{G}_{i, j-12, k}^{(n, n+1)}$, $\bar{H}_{i, j, k+1 / 2}^{(n, n+1)}, \bar{H}_{i, j, k-1 / 2}^{(n, n+1)}$. If these fluxes are computed using the semi-Lagrangian method, it is called the flux-form semiLagrangian scheme (Casulli 1990, 1999; Lin and Rood 1996).

\subsection{Transformation of Temporal Integration into Spa- tial Mean}

For simplicity and no loss of generality, we consider one dimensional problem of Eq. (22) without source/sink term (i.e., $\hat{S}_{i j k}=0$ ),

$\frac{\tilde{\phi}_{i}^{n+1}-\tilde{\phi}_{i}^{n}}{\Delta t}=\frac{\bar{F}_{i+1 / 2}^{(n, n+1)}-\bar{F}_{i-1 / 2}^{(n, n+1)}}{\Delta x}$

From the semi-Lagrangain consideration, we have

$\tilde{\phi}\left(x_{i}^{*}, t_{n}\right)=\tilde{\phi}\left(x_{i}, t_{n}\right)+\frac{\left[\bar{F}_{i+1 / 2}^{(n, n+1)}-\bar{F}_{i-1 / 2}^{(n, n+1)}\right] \Delta t}{\Delta x}$
In the existing flux-form semi-Lagrangian schemes, the temporally integrated flux $\bar{F}_{i=1 / 2}^{(n, n+1)}$ [similar for $\left.\bar{F}_{i+1 / 2}^{(n, n+1)}\right]$ is given by the mean value at the two time steps $t_{n}$ and $t_{n+1}$ (e.g., Casulli 1990; Tanguay et al. 1990),

$\bar{F}_{i-1 / 2}^{(n, n+1)}=\frac{1}{2}\left(F_{i-1 / 2}^{n}+F_{i-1 / 2}^{n+1}\right)$

Using the characteristic-line concept, the flux at time step $t_{n+1}$ and location $x_{i-1 / 2}$ can be transformed into the flux at time step $t_{n}$ and location $x_{i-1 / 2-\mathrm{C}}$ (Fig. 2),

$F_{i-1 / 2}^{n+1}=F_{i-1 / 2-c}^{n}$

Substitution of Eq. (28b) into Eq. (28a) gives

$\bar{F}_{i-1 / 2}^{(n, n+1)}=\frac{1}{2}\left(F_{i-1 / 2}^{n}+F_{i-1 / 2-c}^{n}\right)$

with the mean flux $\bar{F}_{i-1 / 2}^{(n, n+1)}$ determined at the current time step $t_{n}$. Here

$C=\frac{u \Delta t}{\Delta x}$

is the Courant number.

Here, we propose a new method to compute the temporally averaged flux $\bar{F}_{i-1 / 2}^{(n, n+1)}$ with the transformation into spatial averaged flux,

$\bar{F}_{i-1 / 2}^{(n, n+1)} \equiv \frac{1}{\Delta t} \int_{t_{n}}^{t_{n}+\Delta t} F\left(x_{i-1 / 2}, t\right) d t=\frac{1}{C \Delta t} \int_{x_{i-1-12}-c \Delta t}^{x_{i-1 / 2}} F\left(x, t_{n}\right) d x$

Substitution of Eq. (29) into Eq. (30) leads to

$$
\bar{F}_{i-1 / 2}^{(n, n+1)} \approx\left\{\begin{aligned}
\frac{F_{i-1 / 2}^{n}+F_{i-1 / 2-C}^{n}}{2} & \text { if } C \leq \frac{1}{2} \\
\frac{\delta_{1 / 2}\left(F_{i-1 / 2}^{n}+F_{i-1}^{n}\right)}{2}+\frac{\sum_{k=1}^{m-1} \delta_{k}\left(F_{i-k}^{n}+F_{i-k-1}^{n}\right)}{2} & \text { if } \mathrm{C}>\frac{1}{2}
\end{aligned}\right.
$$

where

$m=\left[C-\frac{1}{2}\right], \quad \delta_{1 / 2}=\frac{1}{2 C}, \quad \delta_{k}=\frac{1}{C}, \quad \delta_{m}=1-\delta_{1 / 2}-\sum_{k=1}^{m-1} \delta_{k}$

The bracket [ ] represents the round-off integer. Similarly, the temporally averaged flux at the right boundary $\left(x=x_{i+1 / 2}\right)$ 


$$
\bar{F}_{i+1 / 2}^{(n, n+1)} \approx\left\{\begin{aligned}
\frac{F_{i+1 / 2}^{n}+F_{i+1 / 2-C}^{n}}{2} & \text { if } C \leq \frac{1}{2} \\
\frac{\delta_{1 / 2}\left(F_{i+1 / 2}^{n}+F_{i}^{n}\right)}{2}+\frac{\sum_{k=1}^{m-1} \delta_{k}\left(F_{i-k+1}^{n}+F_{i-k}^{n}\right)}{2} & \text { if } \mathrm{C}>\frac{1}{2}
\end{aligned}\right.
$$

The temporally averaged fluxes $\bar{F}_{i-1 / 2}^{(n, n+1)}$ and $\bar{F}_{i+1 / 2}^{(n, n+1)}$ (from $t_{n}$ to $t_{n}+\Delta t$ ) are transformed into the spatially averaged fluxes over multiple grids at time step $t_{n}$ with weights of $\delta_{1 / 2}, \delta_{1}, \ldots$, $\delta_{m}$. If the characteristic line at $t_{n}$ is beyond the boundary, the boundary condition can be used to calculate $\bar{F}_{i-1 / 2}^{(n, n+1)}$ (Fig. 3),

$\bar{F}_{3 / 2}^{(n, n+1)}=\frac{\frac{1}{2}\left(F_{1}^{n}+F_{3 / 2}^{n}\right)+\left(C-\frac{1}{2}\right)\left(F_{b}^{n}+F_{1}^{n}\right)}{2 C}$

where $F_{b}^{n}$ is the boundary value between $F_{1}^{n}$ and $F_{1}^{n+1}$, and is interpolated by

$F_{b}^{n}=\left(1-\frac{1}{2 C}\right) F_{1}^{n+1}+\frac{1}{2 C} F_{1}^{n}$

Substitution of Eqs. (31) and (33) into the difference Eq. (26) leads to

$\phi_{i}^{n+1}=\phi_{i}^{n}+\left\{\begin{array}{l}-\frac{C}{2}\left(\phi_{i+1}^{n}-\phi_{i-1}^{n}\right)+\frac{C^{2}}{2}\left(\phi_{i+1}^{n}-2 \phi_{i}^{n}+\phi_{i-1}^{n}\right), C \leq \frac{1}{2} \\ -\frac{1}{4}\left(\phi_{i+1}^{n}-\phi_{i-1}^{n}\right)-\frac{1}{2}\left(\phi_{i}^{n}+\phi_{i-1}^{n}-\phi_{i-m}^{n}-\phi_{i-m-1}^{n}\right) \\ -\left(D-\frac{D^{2}}{2}\right)\left(\phi_{i-m}^{n}-\phi_{i-m-1}^{n}\right)-\frac{D^{2}}{2}\left(\phi_{i-m-1}^{n}-\phi_{i-m-2}^{n}\right) \\ \quad+\frac{1}{8}\left(\phi_{i+1}^{n}-2 \phi_{i}^{n}+\phi_{i-1}^{n}\right), C>\frac{1}{2}\end{array}\right.$

which is called the Transformed Flux-formed Semi-Lagrangian (TFSL) scheme for the advection-diffusion Eq. (21). Here, $D=C-m-1 / 2$. The major difference between the existing flux-form semi-Lagrangian scheme and the TFSL scheme comes from the different calculation of the temporally averaged flux $\bar{F}_{i-1 / 2}^{(n, n+1)}$ : Eq. (28c) for the existing fluxform semi-Lagrangian scheme and Eq. (31) for the TFSL scheme.

For $C \leq 2$, the TFSL scheme is the same as the LaxWendroff scheme. Compared to the central difference (CED), the TFSL-scheme has an extra positive term,

TFSL-CED $=\frac{C^{2}}{2}\left(\phi_{i+1}^{n}-2 \phi_{i}^{n}+\phi_{i-1}^{n}\right)$

for $C \leq 1 / 2$. This term can be regarded as the numerical (positive) diffusion which leads to computational stability.

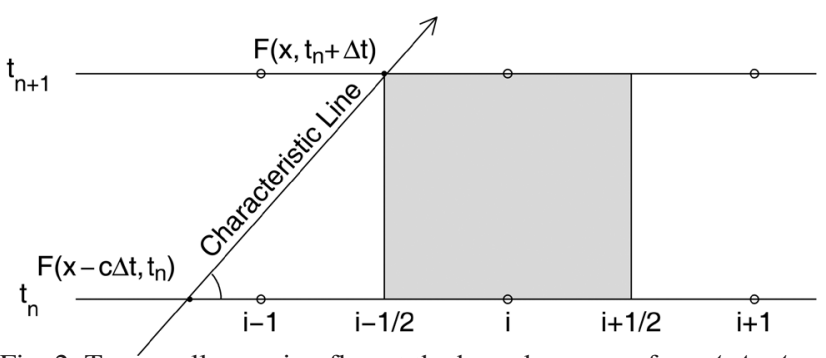

Fig. 2. Temporally varying flux at the boundary $x_{i-1 / 2}$ from $t_{n}$ to $t_{n}+$ $\Delta t$ is transformed into spatially varying flux at $t_{n}$ from $x_{i-1 / 2}-C \Delta t$ to $x_{i-1 / 2}$ using the characteristic-line concept.

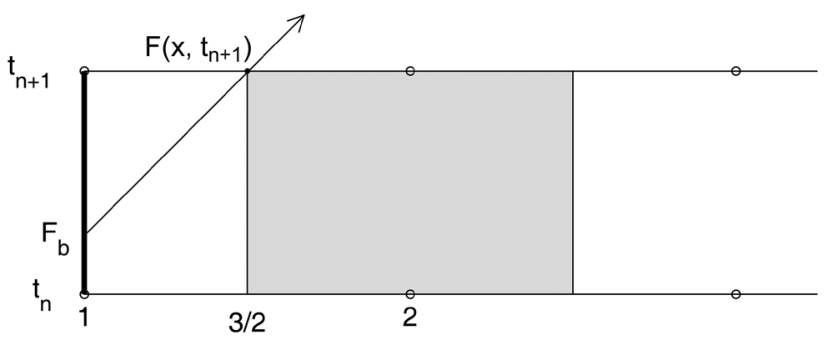

Fig. 3. Same as Fig. 2 except at the left boundary of the integration domain.

Different schemes have different algorithms to compute the temporally averaged fluxes $\bar{F}_{i-1 / 2}^{(n, n+1)}$ and $\bar{F}_{i+1 / 2}^{(n, n+1)}$ (from $t_{n}$ to $t_{n}$ $+\Delta t)$. The TFSL scheme has second order accuracy in both time and space.

\section{STABILITY OF THE TFSL SCHEME}

The stability of numerical schemes is an important issue in solving the advection Eq. (16). In section 3, we showed the instability of the existing schemes (upwind, central, and Lax-Wendroff). To determine the stability of the TFSL scheme Eq. (36), the Fourier series expansion is used. Decay or growth of an amplification factor indicates whether or not the numerical algorithm is stable (von Neumann and Richtmyer 1950). Assuming that at any time step $t_{n}$, the compute solution $\phi_{i}^{n}$ is the sum of the exact solution $\phi_{i}^{n(e x)}$ and error $\varepsilon_{i}^{n}$,

$\phi_{i}^{n}=\phi_{i}^{n(e x)}+\varepsilon_{i}^{n}$

and substituting Eq. (38) into Eq. (36), we obtain

$$
\varepsilon_{i}^{n+1}=\varepsilon_{i}^{n}+\left\{\begin{array}{c}
-\frac{C}{2}\left(\varepsilon_{i+1}^{n}-\varepsilon_{i-1}^{n}\right)+\frac{C^{2}}{2}\left(\varepsilon_{i+1}^{n}-2 \varepsilon_{i}^{n}+\varepsilon_{i-1}^{n}\right), C \leq \frac{1}{2} \\
-\frac{1}{4}\left(\varepsilon_{i+1}^{n}-\varepsilon_{i-1}^{n}\right)-\frac{1}{2}\left(\varepsilon_{i}^{n}+\varepsilon_{i-1}^{n}-\varepsilon_{i-m}^{n}-\varepsilon_{i-m-1}^{n}\right) \\
\quad-\left(D-\frac{D^{2}}{2}\right)\left(\varepsilon_{i-m}^{n}-\varepsilon_{i-m-1}^{n}\right)-\frac{D^{2}}{2}\left(\varepsilon_{i-m-1}^{n}-\varepsilon_{i-m-2}^{n}\right) \\
\quad+\frac{1}{8}\left(\varepsilon_{i+1}^{n}-2 \varepsilon_{i}^{n}+\varepsilon_{i-1}^{n}\right), C>\frac{1}{2}
\end{array}\right.
$$


The finite mesh function, $\varepsilon_{i}^{n}$, can be decomposed into a Fourier series,

$\varepsilon_{i}^{n}=\sum_{j=-N_{x}}^{N_{x}} a_{j}^{n} \exp (I i \theta), \quad \theta=j \pi / N_{x}$

with $I \equiv \sqrt{-1},\left(a_{j}^{n}, \theta\right)$ being the amplitude and phase angle of the jth harmonic. Substituting Eq. (40) into Eq. (39) yields

$a^{n+1}=g(\theta, C) a^{n}$

where

$g(\theta, C)=\left\{\begin{aligned} 1-C^{2}(1-\cos \theta)-I C \sin \theta, \quad C \leq \frac{1}{2} \\ \frac{1}{4}(1-\cos \theta)+\left(\frac{1}{2}-D+\frac{D^{2}}{2}\right) \cos (m \theta) \\ +\left(\frac{1}{2}+D-D^{2}\right) \cos [(m-1) \theta] \\ +\frac{D^{2}}{2} \cos [(m-2) \theta] \\ -I\left\{\left(\frac{1}{2}-D+\frac{D^{2}}{2}\right) \sin (m \theta)\right. \\ +\left(\frac{1}{2}+D-D^{2}\right) \sin [(m-1) \theta] \\ \left.+\frac{D^{2}}{2} \sin [(m-2) \theta]\right\}, \quad C>\frac{1}{2}\end{aligned}\right.$

is called the amplification factor, whose magnitude is given by

$$
|g(\theta, C)|^{2}=\left\{\begin{array}{c}
{\left[1-C^{2}(1-\cos \theta)\right]^{2}+C^{2} \sin ^{2} \theta, \quad C \leq \frac{1}{2}} \\
\frac{1}{16}(1-\cos \theta)^{2}+\left(\frac{1}{2}-D+\frac{D^{2}}{2}\right)^{2} \\
+\left(\frac{1}{2}+D-D^{2}\right)^{2}+\frac{D^{4}}{4} \\
+\frac{1}{2}(1-\cos \theta)\left\{\left(\frac{1}{2}-D+\frac{D^{2}}{2}\right) \cos (m \theta)\right. \\
+\left(\frac{1}{2}+D-D^{2}\right) \cos [(m-1) \theta] \\
\left.+\frac{D^{2}}{2} \cos [(m-2) \theta]\right\} \\
+\left(1-2 D+2 D^{2}\right)\left(\frac{1}{2}+D-D^{2}\right) \cos \theta \\
+D^{2}\left(\frac{1}{2}-D+\frac{D^{2}}{2}\right) \cos (2 \theta), \quad C>\frac{1}{2}
\end{array}\right.
$$

The TFSL-scheme is computationally stable if $|g(\theta, C)| \leq 1$ and computationally unstable if $|g(\theta, C)|>1$. Figure 4 shows that $|g(\theta, C)| \leq 1$ for all $\theta$ and $C$ (larger than 20), which implies that the TFSL-scheme Eq. (36) is stable for all the $C$ values (without Courant number restriction).

\section{SIMULATING THE ROSSBY SOLITON USING THE TFSL SCHEME}

The TFSL-scheme Eq. (36) is only for a spatially variant and temporally invariant $u$. When $u$ [or $-f_{1} \eta$ in Eq. (7)] at $x_{i-1 / 2}$ varies with time from $t_{n}$ to $t_{n+1}$, concept of variant characteristic lines can be used to determine $u\left(x_{i-1 / 2}, t\right)$ with sub time-steps $\left(\delta t_{1 / 2}, \delta t_{1, \ldots}, \delta t_{m}\right)$ (between $t_{n}$ and $\left.t_{n+1}\right)$ from $u\left(x, t_{n}\right)$ at grid points $\left(x_{i-1}, \ldots, x_{i-m}, x_{i^{*}}\right)$, and for $u>0$ the time from the left neighboring grid $x_{i-[k+1]}$ to $x_{i-k}$ is given by (Fig. 5),

$$
\begin{aligned}
\delta t_{k} & =\int_{x_{i-k+1]}}^{x_{i-k}} \frac{d x}{u\left(x, t_{n}\right)}=\frac{\Delta x}{u_{i-k}^{n}} \int_{0}^{1} \frac{d z}{1-\zeta_{1-k} z}=-\frac{\Delta t}{C_{i-k}} \frac{\ln \left(1-\zeta_{i-k}\right)}{\zeta} \\
& =\frac{\Delta t}{C_{i-k}}\left(1+\frac{\zeta_{i-k}}{2}+\frac{\zeta_{i-k}}{3}+\ldots\right), \quad k=0.5,1,2, \ldots, m
\end{aligned}
$$

where

$$
\Delta x=x_{i-k}-x_{i-(k+1)}, \quad \zeta_{i-k}=\frac{u_{i-k}^{n}-u_{i-k+1)}^{n}}{u_{i-k}^{n}}, \quad C_{i-k}=\frac{u_{i-k}^{n} \Delta t}{\Delta x}
$$

The parameter $C_{i-k}$ is the Courant number for sub time steps. A formula similar to Eq. (44) can be obtained for $u<0$ (using the right neighboring grid). The temporally averaged fluxes from $t_{n}$ to $t_{n}+\Delta t$ can be calculated by f taking $\bar{F}_{i-1 / 2}^{(n, n+1)}$ [see Eq. (31)] as the example\}

$$
\begin{aligned}
\bar{F}_{i-1 / 2}^{(n, n+1)}= & \frac{1}{\Delta t}\left[\delta t_{1 / 2} \frac{F_{i-1 / 2}+F_{i-1}}{2}+\delta t_{1} \frac{F_{i-1}+F_{i-2}}{2}+\ldots\right. \\
& \left.+\delta t_{m-1} \frac{F_{i-m+1}+F_{i-m}}{2}+\delta t_{m} \frac{F_{i-m}+F_{i-m-1}}{2}\right]
\end{aligned}
$$

Equation (7) for the Rossby soliton is discretized using the flux form,

$$
\frac{\eta_{i}^{n+1}-\eta_{i}^{n}}{\Delta t}=\frac{\bar{F}_{i+1 / 2}^{(n, n+1)}-\bar{F}_{i-1 / 2}^{(n, n+1)}}{\Delta s}+\hat{S}_{i}
$$

where $\hat{S}_{i}$ is the temporally-spatially averaged source term

$$
\hat{S}_{i} \equiv \frac{1}{\Delta t \Delta s} \int_{t_{n}}^{t_{n+1}+1} \int_{s_{i-1 / 2}}^{s_{i+1 / 2}} S(s, t) d s d t
$$

with $S(s, t)$ given by Eq. (8). The difference, Eq. (47), is solved numerically from the initial condition Eq. (15) using the TFSL-scheme. To compare with the existing flux-form semi-Lagrangian scheme, the Courant number is set to 1.5. 


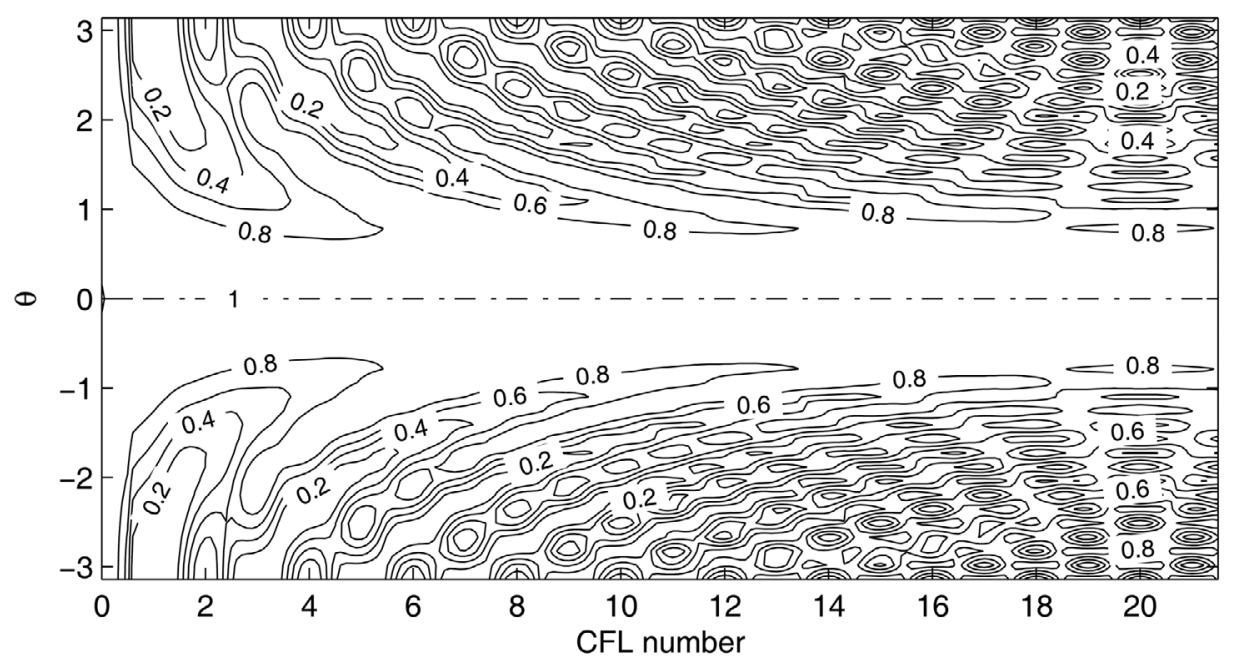

Fig. 4. Dependence of the amplification factor $|g(\theta, C)|$ of the TSFL scheme on $\theta$ and $C$.

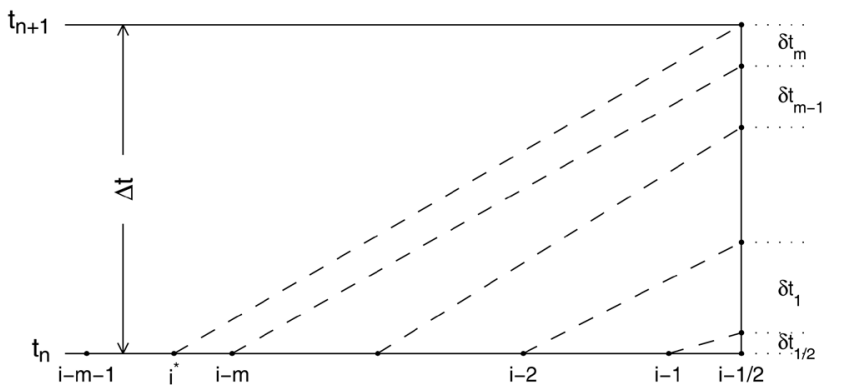

Fig. 5. Same as Fig. 2 except for temporally varying $u$.

After the numerical solution $\eta\left(x_{i}, t_{n}\right)$ is obtained, substituting it into Eq. (4c) yields $\phi\left(s_{i}, y_{i}, t_{n}\right)$ as shown in Fig. 6. Note that the flux-form semi-Lagrangian scheme is highly distorted (Fig. 6) with the numerical solution diverging at $40^{\circ} 30^{\prime} \mathrm{W}$, which may be caused by the error accumulation. However, the TFSL-scheme is quite stable and accurate. After propagating westward around the earth the numerical Rossby soliton (using the TFSL scheme) appears to be almost non-diffusive and non-dispersive.

To show the quality of the TFSL-scheme, the difference Eq. (47) is integrated for $C=1.5$ for a long time period corresponding to the Rossby soliton propagates westward around the earth 5 times. The solution $\phi\left(s_{i}, y_{i}, t_{n}\right)$ is stable all the time (Fig. 7). The relative root-mean-square error (rrmse),

$$
\operatorname{rrmse}(t)=\frac{\sqrt{\frac{1}{\mathrm{~N}_{s} \mathrm{~N}_{y}} \sum_{i=1}^{N_{s}} \sum_{j=1}^{N_{y}}\left[\phi^{(n u m)}\left(\mathrm{s}_{i}, \mathrm{y}_{j}, \mathrm{t}\right)-\phi^{(e x)}\left(\mathrm{s}_{i}, \mathrm{y}_{j}, \mathrm{t}\right)\right]^{2}}}{\max \left|\phi^{(e x)}\left(\mathrm{s}_{i}, \mathrm{y}_{j}, \mathrm{t}\right)\right|}
$$

is calculated to illustrate the accuracy of the TFSL scheme. Table 1 shows RRMSE at the end of first five cycles around the earth. The error varies from $2.66 \%$ for the first cycle to $3.53 \%$ for the fifth cycle.

\section{CONCLUSIONS}

(1) This study shows that the TFSL scheme is a promising stable and accurate method for solving the advectiondiffusion equation. The Fourier analysis shows that the TFSL scheme has second-order accuracy in time and space. This scheme retains the good features of semiLagrangian schemes (no Courant number limitation) with higher accuracy. Computational stability and higher accuracy than the widely used schemes (central, upwind, Lax-Wendroff, semi-Lagragian) makes this technique useful in ocean modeling, computational fluid dynamics, and numerical weather prediction.

(2) Several major features distinguish the TFSL scheme from existing schemes, both Eulerian and semi-Lagrangian. First, the flux $(F)$ at the side of each grid cell is computed not from a single time step (present or next) but from a temporal integration from the present time step to the next time step. Second, this temporal integration is transformed into a spatial integration at the present time step using the characteristic line method.

(3) The equatorial Rossby soliton is used to test the capability of the TFSL scheme since it has exact solution. The equation is solved numerically from the soliton initially located at the equator and $0^{\circ}$ longitude with an overall Courant number of 0.75 . The upwind, central, and Lax-Wendroff schemes greatly distort the Rossby soliton and diverge as it propagates. However, the TFSL scheme does not distort the Rossby soliton and converges as it propagates many cycles around the earth. With an overall Courant number of 1.5 , the numerical Rossby soliton can still propagate many cycles around the earth using the TFSL scheme, but diverges at $40^{\circ} 30^{\prime} \mathrm{W}$ using the flux-form semi-Lagrangian scheme.

(4) Application of the TFSL scheme to the atmospheric and 
(a) Analytic Solution with Courant Number= 1.5
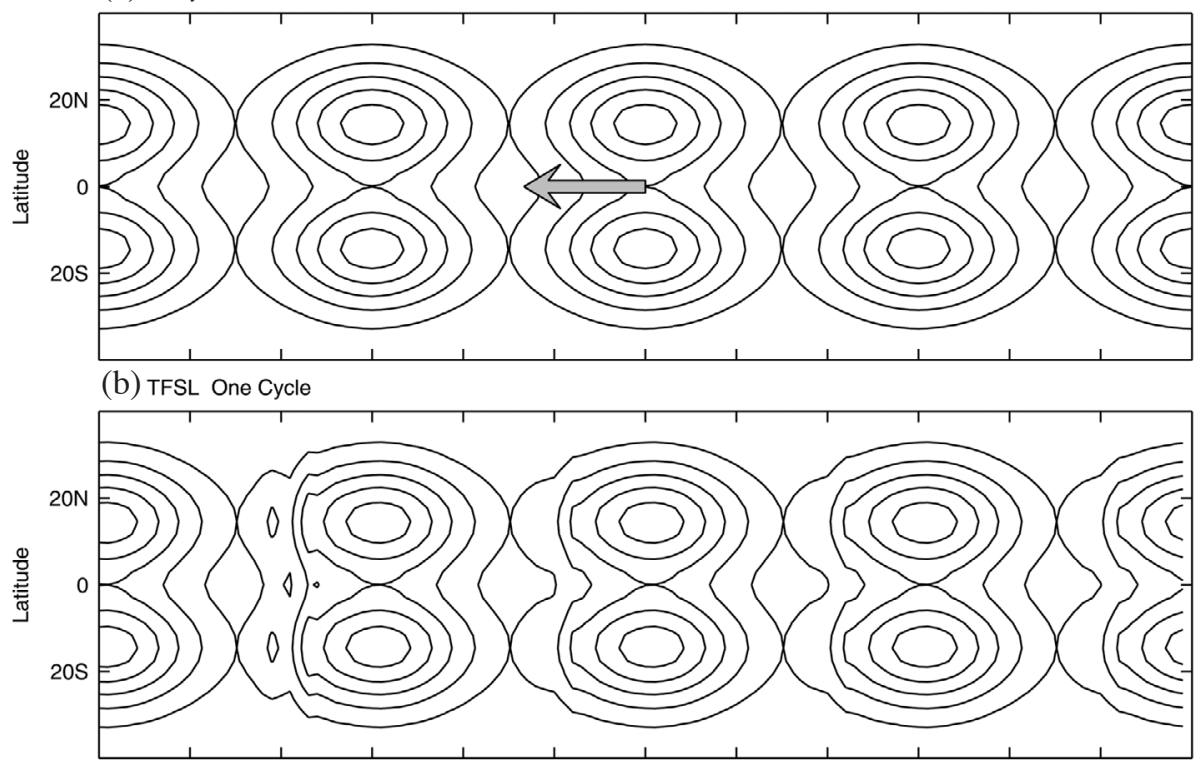

(c) SL diverges at Ion: $40.5 \mathrm{~W}$

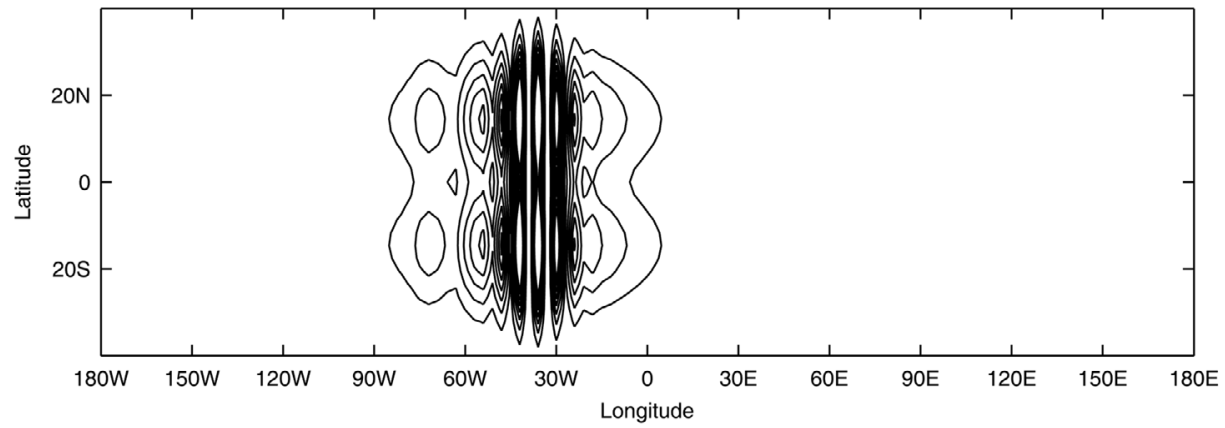

Fig. 6. Surface elevation $\phi(s, y, t)$ of the Rossby soliton obtained from (a) exact solution, and numerical integration with $C=1.5$ using (b) TFSLscheme, and (c) flux-form semi-Lagrangian scheme. The solutions $\phi(s, y, t)$ are plotted at four time instances for the Rossby soliton (exact solution) westward propagating $90^{\circ}, 180^{\circ}, 270^{\circ}$, and $360^{\circ}$ (return to the initial location).
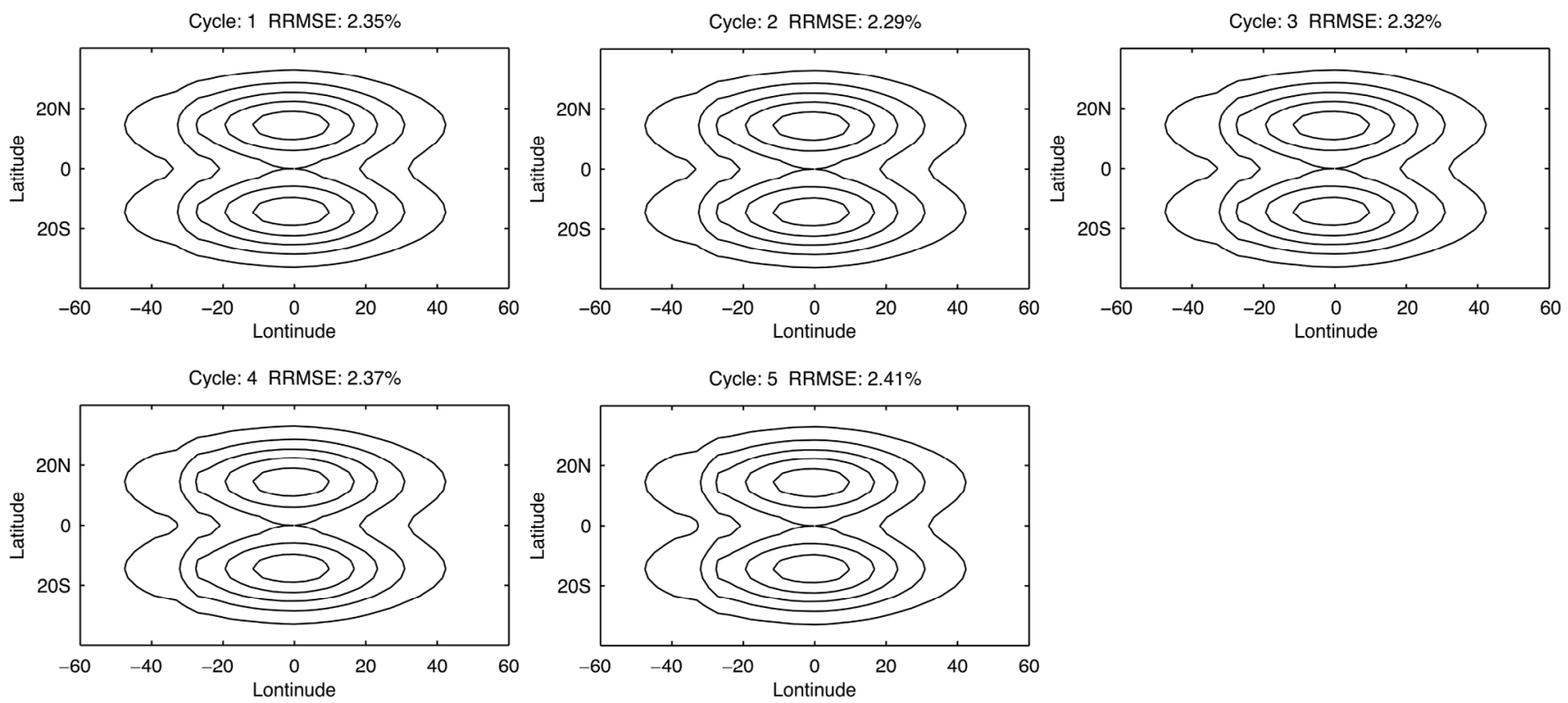

Fig. 7. Surface elevation $\phi(s, y, t)$ of the Rossby soliton after $1-5$ cycles around the earth obtained from numerical integration with $C=1.5$ using the TFSL scheme. 
Table 1. RRMSE of the surface elevation predicted using the TFSLscheme after the first five cycles around the earth.

\begin{tabular}{l|ccccc}
\hline Cycle & 1 & 2 & 3 & 4 & 5 \\
\hline RRMSE (\%) & 2.66 & 2.86 & 3.00 & 3.22 & 3.53 \\
\hline
\end{tabular}

oceanic models needs more research. This is because that the highly accurate treatment of the source term enabled good performance of the TFSL scheme. However, this is a very special case because the analytical solution of S (source term) is known for this system. In ocean modeling, computational fluid dynamics, or numerical weather prediction, source term analytical solutions are usually unknown and thus several iteration processes will be required for the departure/arrival point estimation as well as the source term estimation (for spatial and temporal averaging), which causes the loss of efficiency and accuracy.

(5) The TFSL scheme was developed on the basis of a finite volume approach. It is relatively easy to extend one-dimensional space-time transformation Eqs. (28b) and $(28 \mathrm{c})$ into three-dimensional transformations. The space integration of the flux is conducted over the twodimensional surface of the finite volume, and the time integration is for that volume (see section 4.2). This will be reported in a separate paper in the near future.

Acknowledgements The Office of Naval Research, the Naval Oceanographic Office, and the Naval Postgraduate School supported this study.

\section{REFERENCES}

Boyd, J. P., 1980: Equatorial solitary waves. Part-1: Rossby solitons. J. Phys. Oceanogr., 10, 1699-1717, doi: 10.
1175/1520-0485(1980)010<1699:ESWPIR>2.0.CO;2. [Link]

Casulli, V., 1990: Semi-implicit difference methods for the two-dimensional shallow water equations. J. Comput. Phys., 86, 56-74, doi: 10.1016/0021-9991(90)90091-E. [Link]

Casulli, V., 1999: A semi-implicit finite difference method for non-hydrostatic, free surface flows. Int. J. Numer. Methods Fluids, 30, 425-440, doi: 10.1002/(SICI)10970363(19990630)30:4<425::AID-FLD847>3.0.CO; 2-D. [Link]

Chu, P. C. and C. W. Fan, 1998: A three-point combined compact difference scheme. J. Comput. Phys., 140, 370-399, doi: 10.1006/jcph.1998.5899. [Link]

Chu, P. C. and C. W. Fan, 1999: A three-point non-uniform combined compact difference scheme. J. Comput. Phys., 148, 663-674, doi: 10.1006/jcph.1998.6141. [Link]

Lax, P. and B. Wendroff, 1960: Systems of conservation laws. Commun. Pure Appl. Math., 13, 217-237, doi: 10.1002/cpa.3160130205. [Link]

Lin S. and R. B. Rood, 1996: Multidimensional flux-form semi-Lagrangian transportation schemes. Mon. Weather Rev., 124, 2046-2070, doi: 10.1175/1520-0493(199 6)124<2046:MFFSLT>2.0.CO;2. [Link]

Rood, R. B., 1987: Numerical advection algorithms and their role in atmospheric transport and chemistry models. Rev. Geophys., 25, 71-100, doi: 10.1029/RG025i001p 00071. [Link]

Tanguay, M., A. Robert, and R. Laprise, 1990: A semiimplicit semi-Lagrangian fully compressible regional forecast model. Mon. Weather Rev., 118, 1970-1980, doi:10.1175/1520-0493(1990)118<1970:ASISLF>2.0. CO;2. [Link]

von Neumann, J. and R. D. Richtmyer, 1950: A method for the numerical calculation of hydrodynamic shocks. $J$. Appl. Phys., 21, 232, doi:10.1063/1.1699639. [Link] 